Hirosaki Med. J. 61(Suppl.) : S135-S141, 2010

\title{
PLASMA ANTIBODIES TO Aß40 AND Aß42 IN PATIENTS WITH ALZHEIMER'S DISEASE AND NORMAL CONTROLS
}

\author{
Mikio Shoji ${ }^{1)}$
}

\begin{abstract}
Antibodies to amyloid $ß$ protein $(A ß)$ are present naturally or after $A ß$ vaccine therapy in human plasma. To clarify their clinical role, we examined plasma samples from 113 patients with Alzheimer's disease (AD) and 205 normal controls using the tissue amyloid plaque immunoreactivity (TAPIR) assay. A high positive rate of TAPIR was revealed in AD (45\%) and age-matched controls (41\%), however, no significance was observed. No significant difference was observed in the MMS score or disease duration between TAPIR-positive and negative samples. TAPIR-positive plasma reacted with the Aß40 monomer and dimer, and the Aß42 monomer weakly, but not with the Aß42 dimer. TAPIR was even detected in samples from young normal subjects and young Tg2576 transgenic mice. Although the Aß40 level and Aß40/42 ratio increased, and Aß42 was significantly decreased in plasma from AD groups when compared to controls, no significant correlations were revealed between plasma Aß levels and TAPIR grading. Thus an immune response to $A ß 40$ and immune tolerance to $A ß 42$ occurred naturally in humans without a close relationship to the $A ß$ burden in the brain. Clarification of the mechanism of the immune response to $A ß 42$ is necessary for realization of an immunotherapy for AD.
\end{abstract}

Hirosaki Med. J. 61, Supplement : S135-S141, 2010

\section{Introduction}

Recent studies suggested that Aß immunotherapy is the most promising among the many candidate therapies for AD. Schenk and others showed that an Aß42 peptide vaccine clearly reduced the $A ß$ amyloid burden in transgenic model mice ${ }^{1)}$. Passive immunization using anti$A ß$ antibodies was also shown to be effective for reduction of the $A ß$ amyloid burden ${ }^{2}$. These findings suggest peripheral antibodies to $A ß$ may serve a protective role against AD. A detectable increase in antibodies to Aß42 was observed in about $25 \%$ of patients who received AN1792 in a Phase Istudy ${ }^{3,4}$. Analysis of serum samples by ELISA indicated that 15 of 18 patients experiencing meningoencephalitis in a Phase II study had antibodies against Aß42. CSF antibodies to Aß42 were present in 6 of 8 patients tested after the onset of encephalitis.
However, titers of antibodies to Aß42 were not correlated with the occurrence or severity of symptoms or relapses ${ }^{4}$. An autoantibody to $A ß 40$ was first detected in human $B$ cell lines from AD patients ${ }^{5)}$. Naturally occurring antibodies to synthetic Aß40 were confirmed by ELISA in the CSF and plasma of nonimmunized humans and titers were significantly higher in healthy controls than in patients with $A D^{6}$. Titers of anti-Aß42 peptide antibodies were lower in $\mathrm{AD}$ patients compared with healthy individuals ${ }^{7}$, or elevated in $\mathrm{AD}$ patients and elder transgenic mice ${ }^{8}$. Naturally occurring anti-Aß42 antibodies were detected at very low levels by ELISA in over $50 \%$ of elderly individuals and at modest levels in $5 \%$ of them. Neither the presence nor the amount of naturally occurring anti-Aß42 antibodies correlated with the presence, or age of $\mathrm{AD}$ onset, or the plasma levels of $A ß 40$ and $A ß 42^{9}$. Normal levels of
1) correspondence author

Department of Neurology, Hirosaki University Graduate School of Medicine, 5 Zaifu-cho, Hirosaki, Aomori 036-8562, Japan
Phone: + 81-172-39-5142

FAX: + 81-172-39-5143

E-mail address: mshoji@cc.hirosaki-u.ac.jp 
antibodies to $A ß 42$ and $A ß 40$ were present in both $\mathrm{AD}$ and control groups, even in a young population ${ }^{10)}$. Thus, the previous reports suggested complex relationships for naturally occurring antibodies to $\mathrm{A} \beta$.

Here, we examined 113 AD cases and 155 age-matched normal controls by TAPIR assay in order to clarify the positive rates, antibody characters, correlations with clinical symptoms, and clinical roles of naturally occurring antibodies against ß-amyloid plaques. Modification of plasma $A ß 40$ and $A ß 42$ concentrations by antibodies to $A ß$ was also studied based on age- or ADdependent alterations of plasma $A ß$ levels.

\section{Materials and Methods}

After informed consent was given, blood samples were collected into 0.1\% EDTA from a total of 318 subjects including 113 patients with $\mathrm{AD}$ (AD group) and 205 normal controls (total normal control group: tNC group). As age- matched controls (aNC group), 115 samples from subjects over 43 years old were selected from the tNC group. The clinical diagnosis of AD was based on NINCDS-ADRDA criteria. The clinical severity of $\mathrm{AD}$ was evaluated using the Mini-Mental State Examination (MMS). AD patients were divided into 3 subgroups according to clinical stages: early stage MMS score $>20$, moderate stage MMS score 10 20, advanced stage MMS score $<10$.

Five $\mu \mathrm{m}$ serial paraffin sections of brains from Tg2576 mice or Alzheimer's patients were used. Sections were incubated at $4^{\circ} \mathrm{C}$ overnight with human or mouse plasma diluted with blocking solution (1:100). Sections were then treated with Vectastain Elite ABC kit (Vector, Burlingame, CA). Immunostaining with Ab9204 (1:1,000, antibody to a synthetic Aß peptide) or without the primary antibody were used as positive and negative controls, respectively.

TAPIR findings were classified into 4 levels: negative -, no senile plaque core; weakly positive \pm , senile plaque cores were stained weakly and less than 5 cores were stained in each brain section on a slide; positive,$+ \geq 5$ senile plaque cores were stained clearly in at least one brain section per slide; strongly positive ++ , most senile plaque cores were strongly labeled when compared to Ab9204 immunostaining.

About $2 \mathrm{~g}$ of gray matter of the AD brain was homogenized with 4 volumes of TBS with protease inhibitors $(1 \mu \mathrm{g} / \mathrm{ml}$ Leupeptin, $1 \mu \mathrm{g} / \mathrm{ml}$ TLCK, $0.1 \mu \mathrm{g} / \mathrm{ml}$ Pepstain A, $1 \mathrm{mM}$ phenylmethysulfonyl fluoride and 1mM EDTA), and centrifuged at 100,000g for 1 hour. The resulting pellet was extracted with $10 \mathrm{ml}$ of $10 \%$ SDS in TBS and then with $1 \mathrm{ml}$ of $99 \%$ formic acid (FA). The final supernatant was lyophilized, dissolved with $20 \mu \mathrm{l}$ of $99 \%$ DMSO, and stored at $-80^{\circ} \mathrm{C}$ until use (formic acid soluble amyloid $\mathrm{A} \beta$ fraction: $F A ß)$.

$20 \mu$ l of protein $\mathrm{G}$ agarose was washed 3 times with $1 \mathrm{ml}$ RIPA buffer. Prewashed protein $G$ agarose was mixed with $600 \mathrm{ng}$ synthetic Aß40, $600 \mathrm{ng}$ synthetic $A ß 42$ or 300 ng FAß in $1 \mathrm{ml}$ of RIPA buffer and incubated at room temperature for 30 minutes. After centrifugation, the resulting supernatant was mixed again with $20 \mu$ l of prewashed protein $G$ agarose and $10 \mu \mathrm{l}$ of plasma, incubated at room temperature for 3 hours, and then centrifuged. The pellet was boiled with $1 \times$ NuPage LDS sample buffer containing $0.1 \mathrm{M}$ dithiothreitol for 10 minutes at $70^{\circ} \mathrm{C}$ and separated on a 4 to $12 \%$ NuPage Bis-Tris gel (Invitrogen, CA). After electro-transfer, the blot membrane was incubated with monoclonal 6E10 (specific to Aß116, 1:1000) at $4^{\circ} \mathrm{C}$ overnight. After washing and incubation with horseradish- peroxidaseconjugated goat anti-mouse IgG (1:2000) at RT for 2 hours, the signal was developed by SuperSignal west Dura extended duration substrate (Pierce Biotechology, CA).

Sandwich ELISA was used to specifically quantify whole plasma Aß, as previously 
described. Microplates were pre-coated with monoclonal BNT77 (IgA, anti-Aß11-28, specific Aß11-16) and sequentially incubated with $100 \mu \mathrm{l}$ of samples followed by horseradish- peroxidaseconjugated BA27 (anti-Aß1-40, specific Aß40) or BC05 (anti-Aß35-43, specific Aß42 and Aß43).

\section{Results}

In the $\mathrm{AD}$ group, 42 cases (37.2\%) were TAPIR -, $20(17.8 \%)$ were, $\pm 44(38.9 \%)$ were grading + , and $7(6.2 \%)$ were ++. Fifty one of 113 $\mathrm{AD}$ patients were ++ and + , suggesting frequent appearance $(45.1 \%)$ of naturally occurring antibodies to amyloid plaque cores. In the aNC group, 54 cases (34.8\%) were TAPIR -, 37 (23.9\%) were \pm , $44(28.4 \%)$ were + , and $20(12.9 \%)$ were ++. Sixty-four cases of 155 aNC group (41.3\%) were TAPIR ++ or + . No significant differences were detected by Mann-Whitney's U tests in the positive rates of naturally occurring antibodies to amyloid plaque cores among groups $(\mathrm{p}=0.77)$, or comparisons between the positive AD group (++ and + ), negative $\mathrm{AD}$ group ( \pm and - ), positive aNC group $(++$ and +$)$ and negative aNC $( \pm$ and -) group $(\mathrm{p}=0.54)$.

There were no significant differences in gender or mean age in both $\mathrm{AD}$ and aNC groups. No significant differences were observed in MMS scores and disease duration among the TAPIR,,,- \pm+++ subgroups of AD samples. There were also no significant differences in the progressive decline of MMS scores among these AD subgroups. The presence of naturally occurring antibodies to $A ß$ as detected by TAPIR may therefore not improve prognosis of AD.

As indicated in Fig 1, freshly prepared Aß40 and $A ß 42$ were composed of monomers and dimers. However, formic acid extractable $A B$ (FAß) exhibited polymerization as shown by the higher molecular mass of its oligomers (Fig 1, left panel). Immunoprecipitation with TAPIR $++/+$ plasma obtained from the $\mathrm{AD}$ and aNC groups retrieved Aß40 monomers and dimers as well as higher molecular mass polymers. Immunodetection of monomeric Aß42 using 6E10 was very weak, whereas no dimeric form of Aß42 was detected (Fig 1 right panels). These findings suggest that TAPIR- positive plasma reacts with $A ß$, but its reactivity to $A ß 42$ is very weak.

In order to clarify when these antibodies against $A ß$ appear, we additionally examined the remaining 50 plasma samples from subjects younger than 43 yeas old in the tNC group. Surprisingly, TAPIR revealed that antibodies to $A ß$ appeared in a 2 year-old child and also in some young subjects (TAPIR +; Fig 2A, $\mathrm{B}$ and $\mathrm{C})$. TAPIR positive rates were $57 \%$ by 10 years old $(n=7 ; 4$ TAPIR +$), 64 \%$ by 20 years old $(\mathrm{n}=11 ; 6$ TAPIR + ), $20 \%$ by 30 years old $(n=10 ; 2$ TAPIR + ) and $10 \%$ by 40 years old $(n=10 ; 1$ TAPIR +$)$. To confirm further this early appearance of antibodies to $A \beta$, immunoprecipitation was performed. Essentially identical finding to those seen in the $\mathrm{AD}$ and aNC groups were revealed (Fig 2D F). Aß40 and FAß monomers and dimers were strongly immunoprecipitated (arrows). However, immunoprecipitation of the Aß42 monomer was also weak and the Aß42 dimer was absent in TAPIR-positive plasma from younger controls.

Plasma from younger and older Tg2576 mice labeled amyloid cores in AD brains (Fig 2G I). The appearance rate was $1 / 3$ at 4 months old (1 TAPIR + ), $3 / 3$ at 8 months old (1 TAPIR ++ and 2 TAPIR + ), $1 / 1$ at 16 months old (1 TAPIR ++ ) and $1 / 1$ at 23 months old mice (1 TAPIR+). Finally, we summarized age- dependent TAPIRpositive rates (TAPIR grading + and ++ ) in 10 year increments in both $\mathrm{AD}$ and $\mathrm{tNC}$ groups (Fig $2 J)$. TAPIR-positive rates were high in young subjects ( $1 \sim 20$ years old), low during adulthood (21 50 years old) and then increased again after 50. No differences were observed between $A D$ and tNC samples from 50 to 91 years old. Thus, 
the appearance of antibodies to $A ß$ preceded $A ß$ amyloid deposition in human and model mouse brains.

To examine the effect of antibodies to $A ß$ on plasma $A ß$ concentrations, we measured levels of $A ß 40$ and $A ß 42$ in 318 plasma samples by specific ELISA. In the tNC group, plasma Aß40 levels increased after 40 years of age (Fig 3A; $\mathrm{P}<0.0001$ ). On the contrary, plasma Aß42 levels increased between the teens and twenties, then gradually declined with age (Fig 3B; $\mathrm{P}=0.0158$ ). The $A ß$ ratio (Aß40/Aß42) was stable until $\sim 30$ years old and then gradually increased (Fig 3C; $\mathrm{P}<0.0001$ ).

Significantly increased levels of plasma Aß40 were observed in the AD group $(112 \pm 39.51$ $\mathrm{pmol} / \mathrm{L})$ compared to the aNC group (95.38 \pm 32.30; $\mathrm{p}<0.0002$; Fig 3D). Aß42 levels were significantly decreased in the AD group (10.29

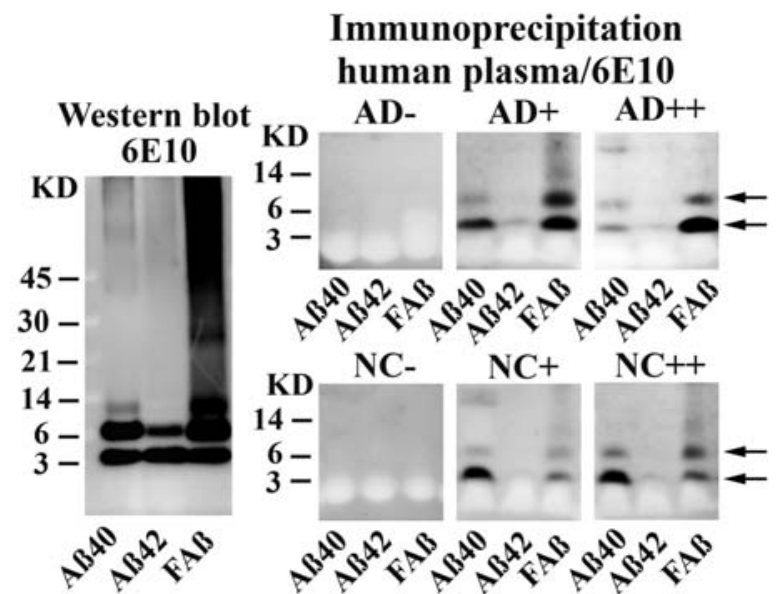

Figure 1 TAPIR-positive plasma immunoprecipitated $A ß 40$ and amyloid $A ß$, but $A ß 42$ very weakly. On direct western blotting of synthetic Aß40, $\mathrm{A} 342$, and $\mathrm{FA}$ (rom the $\mathrm{AD}$ brain, antibody $6 \mathrm{E} 10$ detected monomers and dimers of $A ß 40, A ß 42$ and brain amyloid $A ß$ with smear aggregates (left panel). Immunoprecipitations of $A ß 40, A ß 42$, and FAß using TAPIR-, +, and ++ plasma from the AD group (right upper panel, $\mathrm{AD}$ ) or the aNC group (right lower panel, NC) were labeled by antibody $6 \mathrm{E} 10$, showing that monomers (arrow) and dimers (arrow) of Aß40 were recognized by TAPIR positive plasma (grading + and ++ ) in addition to Aß42 monomers, and brain Aß amyloid monomers and dimers with smear aggregates, which showed weak signals. "Adopted from reference 17." $\pm 13.80 \mathrm{pmol} / \mathrm{L})$ compared to the aNC group $(12.13 \pm 12.29$; $\mathrm{p}<0.0001$; Fig $3 \mathrm{E})$. Based on these changes, the $A ß$ ratio ( $A ß 40 / A ß 42)$ was more strongly increased in the $\mathrm{AD}$ group (14.42 $\pm 10.00)$ than in the aNC group (8.34 \pm 3.83 ; $\mathrm{p}<0.0001$; Fig $3 \mathrm{~F})$. ROC analysis of the $\mathrm{A} \beta$ ratio indicated that the significant cut off value was

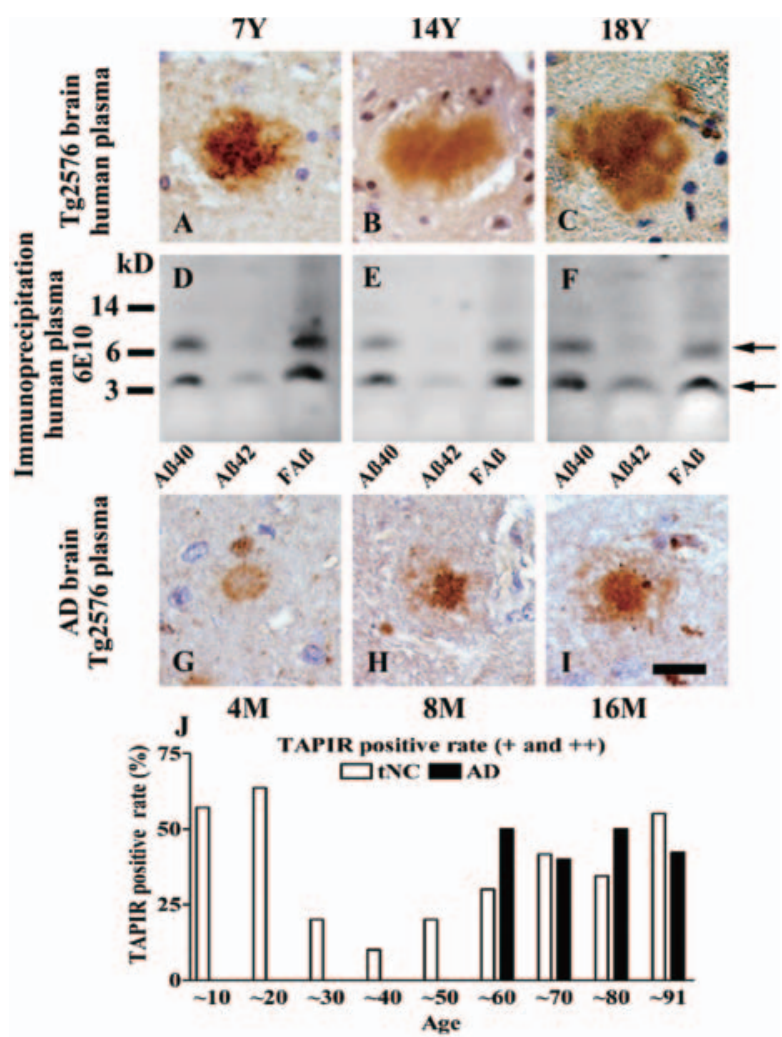

Figure 2 Antibodies to $A ß$ appeared before $A ß$ amyloid deposits in the brain

TAPIR was positive in 7 year old (TAPIR +; A 7Y), 14 year old (TAPIR +; B, 14Y), and 18 year old young persons (TAPIR $+, \mathrm{C}, 18 \mathrm{Y}$ ). TAPIR positive plasma strongly immunoprecipitated monomers and dimers (arrow) of $A ß 40$ and FAß, and weakly immunoprecipitated monomers of $A ß 42$ and $A ß$ amyloid (D, E and F; corresponding plasma of upper panels; D and A 7Y, E and B 14Y and $\mathrm{F}$ and $\mathrm{C} 18 \mathrm{Y}$ ). Plasma from younger and older Tg2576 mice also labeled amyloid cores in AD brains (G: 4 months old TG; H: 8 months old $\mathrm{Tg}$ and I: 16 months old $\mathrm{Tg}$ ). Bar scale $=15 \mu \mathrm{m}$. $\mathrm{J}$ : TAPIR positive rates in the tNC group according to age. Columns show the TAPIR-positive rate (TAPIR grading + and ++ ) for 10 year increases in the AD (black columns) and tNC (white columns) groups. TAPIR-positive rates were high in young subjects (1 20 years old), low during adulthood (21 50 years old) and then increased again after age 50. No differences were observed between $A D$ and $\mathrm{tNC}$ groups in samples from subjects 50 to 91 years old. "Adopted from reference 17." 
9.0, which provided high sensitivity (78.8\%) and low specificity (30.3\%) for clinical diagnosis of AD. When the mean +2 SD (15.9) of the aNC group was used as a cutoff value, the sensitivity was $24 \%$ and the specificity was $96 \%$. When AD was divided into 3 subgroups according to clinical stage, increasing Aß40 levels and $A ß$ ratio, as well as decreasing Aß42 levels progressed from the early stage to the advanced stage (Fig 3G I).

\section{Discussion}

In our study, a high positive rate of TAPIR was found in both $\mathrm{AD}$ and $\mathrm{aNC}$ groups, but no significant difference was found between these groups. Essentially the same findings were observed even in strongly positive $(++)$ subgroups of $\mathrm{AD}$ and aNC. Non-parametric analysis revealed that neither MMSE score nor disease duration correlated with TAPIR grade, indicating that the physiological impact of naturally occurring anti-Aß antibodies is below clinical significance. Our immunoprecipitation study suggested that TAPIR ++/+ plasma obtained from $\mathrm{AD}$ and aNC subjects retrieved Aß40 monomers and dimers as well as higher
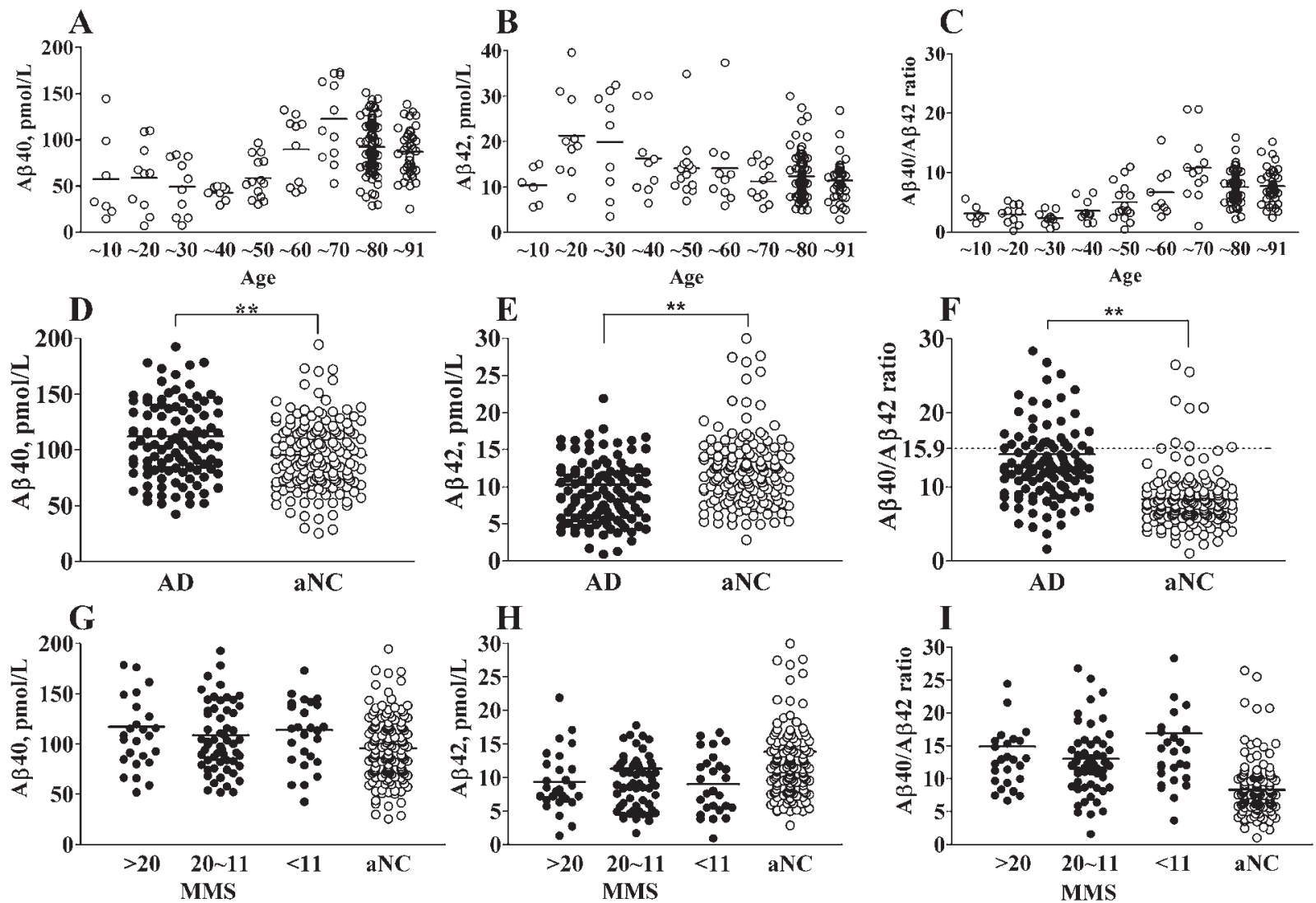

Figure 3 Age-dependent regulation of plasma Aß levels in controls, and their alteration in AD.

Plasma $A ß 40$ and $A ß 42$ levels showed different age-dependent alterations in the $\mathrm{tNC}$ group. $A ß 40$ levels increased from age 50 and decreased from age $70(\mathrm{~A})$. A 442 levels were high in the teens and twenties, then gradually decreased with age (B). Based on these different changes, the $A ß$ ratio (Aß40/Aß42) progressively increased from age 40 (C). Significantly increased levels of $A ß 40(D: p=0.0002)$ and increased $A ß$ ratio $(F: p<0.0001)$ as well as decreased levels of $\mathrm{A} \beta 42(\mathrm{E}: \mathrm{p}<0.0001)$ were shown between the $\mathrm{AD}$ and aNC groups. When the mean $+2 \mathrm{SD}$ of the $\mathrm{A} \beta$ ratio in the aNC group was used as a diagnostic marker for AD, the cut off value 15.9 (dot line) provided $24 \%$ sensitivity and $96 \%$ specificity $(\mathrm{F})$. Constant alterations of plasma $\mathrm{A} ß$ levels in $\mathrm{AD}$ were recognized at the early (MMS score $>20)$, moderate (MMS score 20 11), and advanced stages (MMS score <11) (G I). A, D, G: Aß40; B, E, H: Aß42; C, F, I: Aß ratio. Bars show mean levels. "Adopted from reference 17." 
molecular mass polymers. Immunodetection of monomeric Aß42 using 6E10 was very weak, whereas no dimeric form of Aß42 was detected under our testing conditions. The absence of anti-Aß42 dimer antibodies and the relatively low levels of anti-Aß42 monomers were characteristic of naturally occurring antibodies to Aß. These findings are considered to be another reason why naturally occurring antibodies to $A ß$ are not sufficient for prevention of development of dementia.

Our TAPIR assay also showed that anti-Aß antibodies were naturally present throughout the entire human life span. It is relevant to note that naturally occurring anti-Aß antibodies were unequivocally detected in young human subjects as well as young Tg2576 mice. In relative terms, the positive rates of anti-Aß antibodies were highest in young individuals, lowest in those middle-aged and higher in the elderly. The presence of anti-Aß antibodies in young human subjects was characterized by the subsequent immunoprecipitation study. Anti-Aß antibodies retrieved Aß40 monomers and dimers as well as high molecular mass oligomers in FAß fractions, but they retrieved fewer Aß42 dimers. To our knowledge, this is the first report showing the relatively selective presence of anti-Aß40 antibodies, and reduced amounts of anti-Aß42 antibodies in young individuals.

The exact mechanism underlying spontaneous anti-Aß antibody production remains unknown. Although increased Aß42 levels have been detected in transgenic animal models ${ }^{11)}$, immune hyporesponsiveness to $\mathrm{A} \beta 42$ was also shown ${ }^{12}$. Increased $\mathrm{T}$ cell reactivity to $\mathrm{A} ß 42$ was shown to increase in elderly individuals and patients with $\mathrm{AD}^{13}$. However, the previous findings and our results could not show increased titers of anti-Aß42 antibodies in these groups. Thus, hyopoimmunue responses to $A ß 42$, especially to the Aß42 oligomer, actually occurred in $\mathrm{AD}$ and healthy populations. Since Aß42 is highly pathogenic and neurotoxic, Aß42 may be sequestered and spontaneous immune responses to $A ß$ may be suppressed in human populations. Significantly increased levels of plasma Aß40, increased $A B$ ratio and decreased levels of $A ß 42$ were revealed in the $\mathrm{AD}$ group when compared to the aNC group. When AD was divided into 3 groups according to clinical stage, the $A ß$ ratio increased progressively from the early stage to the advanced stages of AD. These findings show that plasma $A ß$ ratio can be used as an easy, non-invasive, and useful biomarker for diagnosis and monitoring of clinical symptoms of $\mathrm{AD}$, although the sensitivity and specificity are lower than those in CSF samples ${ }^{14-16)}$.

\section{References}

1) Schenk D, Barbour R, Dunn W, Gordon G, Grajeda H, Guido T, Hu K, Huang J, Johnson-Wood K, Khan K, Kholodenko D, Lee M, Liao Z, Lieberburg I, Motter R, Mutter L, Soriano F, Shopp G, Vasquez N, Vandevert C, Walker S, Wogulis M, Yednock T, Games D, Seubert P. Immunization with amyloid- $ß$ attenuates Alzheimer-diseaselike pathology in the PDAPP mouse. Nature 1999;400:173-7.

2)Bard F, Cannon C, Barbour R, Burke RL, Games D, Grajeda H, Guido T, Hu K, Huang J, Johnson-Wood K, Khan K, Kholodenko D, Lee M, Lieberburg I, Motter R, Nguyen M, Soriano F, Vasquez N, Weiss K, Welch B, Seubert P, Schenk D, Yednock T. Peripherally administered antibodies against amyloid ß-peptide enter the central nervous system and reduce pathology in a mouse model of Alzheimer disease. Nat Med 2000;6:916-9.

3) Orgogozo, JM, Gilman S, Dartigues JF, Laurent B, Puel, M, Kirby LC, Jouanny P, Dubois B, Eisner L, Flitman S, Michel BF, Boada M, Frank A, Hock C. Subacute meningoencephalitis in a subset of patients with AD after Aß42 immunization. Neurology 2003;61:46-54.

4) Nicoll JA, Wilkinson D, Holmes C, Steart P, Markham H, Weller RO. Neuropathology of human Alzheimer disease after immunization with amyloid-ß peptide: a case report. Nat Med 
2003;9:448-52.

5) Gaskin F, Finley J, Fang Q, Fu SM. Human antibodies reactive with ß-amyloid protein in Alzheimer's disease. J Exp Med 1993;177:1181-6.

6) Du Y, Dodel R, Hampel H, Buerger K, Lin S, Eastwood B, Bales K, Gao F, Moeller HJ, Oertel W, Farlow M, Paul S. Reduced levels of amyloid ß-peptide antibody in Alzheimer disease. Neurology 2001;57:801-5.

7) Weksler ME, Relkin N, Turkenich R, LaRusse S, Zhou L, Szabo P. Patients with Alzheimer disease have lower levels of serum anti-amyloid peptide antibodies than healthy elderly individuals. Exp Gerontol 2002;37:943-8.

8) Nath A, Hall E, Tuzova M, Dobbs M, Jons M, Anderson C, Woodward J, Guo Z, Fu W, Kryscio R, Wekstein D, Smith C, Markesbery WR, Mattson MP. Autoantibodies to amyloid ß-peptide (Aß) are increased in Alzheimer's disease patients and $A ß$ antibodies can enhance $A ß$ neurotoxicity: implications for disease pathogenesis and vaccine development. Neuromolecular Med 2003;3:29-39.

9) Hyman BT, Smith C, Buldyrev I, Whelan C, Brown H, Tang MX, Mayeux R. Autoantibodies to amyloid- $\beta$ and Alzheimer's disease. Ann Neurol 2001;49:808-10.

10) Baril L, Nicolas L, Croisile B, Crozier P, Hessler C, Sassolas A, McCormick JB, Trannoy E. Immune response to $A ß$-peptides in peripheral blood from patients with Alzheimer's disease and control subjects. Neurosci Lett 2004;355:226-30.

11) Kawarabayashi T, Younkin LH, Saido TC, Shoji M, Ashe KH, Younkin S.G. Age-dependent changes in brain, CSF, and plasma amyloid ß protein in the Tg2576 transgenic mouse model of Alzheimer's disease. J Neurosci 2001;21:372-81.

12) Monsonego A, Maron R, Zota V, Selkoe DJ, Weiner HL. Immune hyporesponsiveness to amyloid $ß$-peptide in amyloid precursor protein transgenic mice: implications for the pathogenesis and treatment of Alzheimer's disease. Proc Natl Acad Sci USA 2001;98:10273-8.

13) Monsonego A, Zota V, Karni A, Krieger JI, BarOr A, Bitan G, Budson AE, Sperling R, Selkoe DJ, Weiner HL. Increased $T$ cell reactivity to amyloid $ß$ protein in older humans and patients with Alzheimer disease. J Clin Invest 2003;112:415-22.

14)Kanai M, Matsubara E, Isoe K, Urakami K, Nakashima K, Arai H, Sasaki H, Abe K, Iwatsubo T, Kosaka T, Watanabe M, Tomidokoro Y, Shizuka M, Mizushima K, Nakamura T, Igeta Y, Ikeda Y, Amari M, Kawarabayashi T, Ishiguro K, Harigaya Y, Wakabayashi K, Okamoto K, Hirai S, Shoji M. Longitudinal study of cerebrospinal fluid levels of tau, Aß1-40, and Aß1-42(43) in Alzheimer's disease: a study in Japan. Ann Neurol 1998;44:17-26.

15) Shoji M, Kanai M, Matsubara E, Tomidokoro Y, Shizuka M, Ikeda Y, Ikeda M, Harigaya Y, Okamoto K, Hirai S. The levels of cerebrospinal fluid $A ß 40$ and $A ß 42(43)$ are regulated agedependently. Neurobiol Aging 2001;22:209-15.

16) Shoji M. Cerebrospinal fluid $A ß 40$ and $A ß 42$ : natural course and clinical usefulness. Front Biosci 2002;7:d997-1006.

17) Xu W, Kawarabayashi T, Matsubara E, Deguchi K, Murakami T, Harigaya Y, Ikeda M, Amari M, Kuwano R, Abe K, Shoji M. Plasma antibodies to Abeta40 and Abeta42 in patients with Alzheimer's disease and normal controls. Brain Res 2008;1219:169179 . 